\title{
Intenção de amamentar entre gestantes: associação com trabalho, fumo e experiência prévia de amamentação
}

\author{
Intention to breastfeed among pregnant women: association with \\ work, smoking, and previous breastfeeding experience
}

Renata Cordeiro Fernandes (https://orcid.org/0000-0002-5278-616X) ${ }^{1}$

Doroteia Aparecida Höfelmann (https://orcid.org/0000-0003-1046-3319) ${ }^{1}$

${ }^{1}$ Programa de Pós-

Graduação em Alimentação e Nutrição, Universidade

Federal do Paraná.

Av. Prefeito Lothario

Meissner 632, Jardim

Botânico. 80210-170

Curitiba PR Brasil.

renatacordeirofernandes@

gmail.com

\begin{abstract}
The objective of this study was to estimate the prevalence of intention to breastfeed (IB) for an insufficient (under 6 months) or prolonged (24 months and longer) amount of time and to investigate its association with demographic and socioeconomic status, health behaviors, obstetric history, and previous breastfeeding experience among pregnant women. This is a cross-sectional study made with pregnant women under prenatal care in 17 units of the Family Health Strategy, in Colombo (PR). Crude and adjusted multinomial logistic regression analyses were used to identify associations between IB and exposure variables. Among pregnant women participating in the survey $(n=316), 99.1 \%$ reported $I B$. The average $I B$ time was 13.5 months. The IB for insufficient and prolonged time was referred to by $9.8 \%$ and $22.0 \%$ of participants respectively. Those who presented the greatest changes of IB for insufficient time were women who: did not have a partner (OR 3.23 , 95\% CI 1.31; 7.94), who performed paid work (OR 5.56, 95\% CI 2.10; 14.71), and smokers (OR 7.79, 95\% CI 2.35; 25.81). Prolonged IB was more frequent among pregnant women with previous experience in prolonged breastfeeding ( $O R$ 3.05, 95\% CI 1.02; 9.03). Factors associated to IA were found to support actions directed to vulnerable groups aiming the promotion of breastfeeding practices.
\end{abstract}

Key words Intention, Breastfeeding, Pregnancy
Resumo O objetivo do presente estudo foi estimar a prevalência de intenção de amamentar (IA) por tempo insuficiente (inferior a 6 meses) ou prolongado (24 meses ou mais) e investigar sua associação com variáveis demográficas, socioeconômicas, comportamentais relacionadas à saúde, antecedentes obstétricos e experiência prévia com amamentação entre gestantes. Estudo transversal com gestantes em acompanhamento pré-natal em 17 unidades da Estratégia Saúde da Família, em Colombo (PR). Análises de regressão logística multinomial brutas e ajustadas foram empregadas para identificar associações entre IA e variáveis de exposição. Dentre as gestantes participantes da pesquisa $(n=316), 99,1 \%$ relataram IA. O tempo médio de IA foi de 13,5 meses. A IA por tempo insuficiente e prolongado foi referida por $9,8 \%$ e $22,0 \%$ das gestantes, respectivamente. Apresentaram maiores chances de IA por tempo insuficiente aquelas que não possuíam companheiro (OR 3,23, IC95\% $1,31 ; 7,94)$, que exerciam trabalho remunerado (OR 5,56, IC95\% 2,10; 14,71) e que eram fumantes (OR 7,79, IC95\% 2,35; 25,81). A IA prolongada foi mais frequente entre as gestantes com experiência prévia em amamentação prolongada (OR 3,05, IC95\% de 1,02; 9,03). Por fim, identifica-se que os fatores associados à IA subsidiam ações voltadas para os grupos vulneráveis com vistas à promoção da prática do aleitamento materno.

Palavras-chave Intenção, Aleitamento materno, Gravidez 


\section{Introdução}

O aleitamento materno contribui positivamente para o desenvolvimento das potencialidades humanas, especialmente durante os primeiros anos de vida. Os benefícios do aleitamento materno transcendem suas qualidades nutricionais e alcançam aspectos imunológicos e sociais a curto e longo prazo para a mãe e o filho ${ }^{1,2}$.

A recomendação da Organização Mundial de Saúde (OMS) é de que o aleitamento materno deve ser mantido até os dois anos ou mais, e complementado com outros alimentos apenas a partir dos seis meses de idade ${ }^{3-5}$.

Em metanálise, na qual foram analisados dados de 153 países, observou-se que os lactentes que foram alimentados ao seio apresentaram menor chance de morbidade e mortalidade ${ }^{6}$. Dentre os benefícios observados às crianças amamentadas por maior tempo, destacam-se: a menor prevalência de diabetes tipo 2 e de excesso de peso; prevenção da má oclusão dentária; e maior inteligência, quando comparadas às crianças amamentadas brevemente ou não amamentadas $^{2,6}$. Para as lactantes, a amamentação pode prevenir o câncer de mama e ovários, aumentar o intervalo entre gestações e reduzir o risco de desenvolver diabetes ${ }^{2}$.

No Brasil, dados de 1999 até 2008 apontaram a ocorrência do desmame precoce e estimaram que a probabilidade de crianças estarem em aleitamento materno aos seis meses de vida era de $77,6 \%$, e aos 12 meses era de $45,5 \%$. Para a região sul do país, essa probabilidade era de 72,1\% aos seis meses e 37,9\% aos 12 meses de vida ${ }^{7}$. Em 2006, a Pesquisa Nacional de Demografia e Saúde da Criança e da Mulher (PNDS) $)^{8}$ constatou que apenas 4 a cada 10 crianças $(39,8 \%)$ com até 180 dias de vida estavam em Aleitamento Materno Exclusivo (AME).

A decisão sobre o tempo de amamentação ocorre antes da gestação, ou ainda no primeiro trimestre da gravidez ${ }^{9}$. A intenção de amamentar (IA) gerada no período pré-natal é um importante fator preditor da duração do aleitamento materno em mulheres que ganharam seus bebês a termo ou pré-termo ${ }^{10,11}$. Dentre as motivações comumente citadas pelas gestantes para amamentar, destacam-se, primeiramente, os benefícios para a saúde da criança, seguidos da naturalidade da amamentação e do fortalecimento do vínculo mãe e filho?.

Uma revisão sistemática sobre a IA verificou que primiparidade, maior idade e escolaridade materna, experiência prévia com amamentação, ausência do uso do cigarro e residir com companheiro colaboraram positivamente para a IA ${ }^{11}$.

Considerando que a intenção prévia do tempo de amamentação influencia a duração real do aleitamento materno ${ }^{9}$, conhecer os fatores relacionados a esta intenção pode ser oportuno para se adotar ações de promoção da amamentação efetivas. Esta pesquisa teve como objetivo estimar a prevalência de intenção de amamentar por tempo insuficiente (inferior a 6 meses) ou prolongadamente (24 meses ou mais) e investigar sua associação com variáveis demográficas, socioeconômicas, comportamentos relacionados à saúde, antecedentes obstétricos e experiência prévia com amamentação entre gestantes.

\section{Métodos}

Este é um estudo transversal, com coleta de dados no período de abril a novembro de 2016, em Colombo, município da região metropolitana de Curitiba, no Paraná (PR). Com população em torno de 234.941 habitantes ${ }^{12} \mathrm{e}$ a Atenção Primária à Saúde composta por 17 Unidades de Saúde da Família (USF), em maio de 2016, o município abrangia 1.375 gestantes em acompanhamento de pré-natal no Sistema Único de Saúde (SUS), segundo estimativas da Secretaria Municipal de Saúde.

Constituem população do estudo as gestantes que realizavam atendimento pré-natal com risco habitual nas USF do município. Para definição da amostra, utilizou-se nível de confiança de $95 \%$, cinco pontos percentuais de margem de erro e considerou-se a prevalência desconhecida do desfecho de $50 \%$, com intenção de aumentar o tamanho da amostra, assim resultando uma amostra mínima de 301 gestantes. A fim de restituir eventuais perdas devidas a recusas, acrescentou-se $20 \%$ no número da amostra, totalizando assim amostra final de 361 gestantes.

A estimativa para estudos de associação a partir da amostra mínima prevista no cálculo de prevalência $(n=301)$ permitiria, mantido o nível de confiança de $95 \%$ e poder de $80 \%$, identificar associações entre desfecho e exposições com uma razão de prevalência mínima de 1,44 , considerando prevalência do desfecho de 38,3\% entre não expostos e de $55 \%$ entre expostos. Os guias para estimativa do tamanho de amostra para regressões multinominais indicam o mínimo de 10 casos para cada variável independente ${ }^{13}$. No presente estudo, o modelo final contou com sete variáveis independentes, distribuídas em 18 cate- 
gorias (o que resultaria em uma amostra mínima de 180 observações).

A amostra foi proporcionalmente distribuída em relação ao número de gestantes cadastradas em cada USF. Todas as gestantes foram convidadas consecutivamente a partir da agenda de consultas pré-natal estabelecida no serviço de saúde. Para participação na pesquisa, utilizaram-se os seguintes critérios de inclusão: estar grávida no momento da entrevista (independentemente da idade gestacional), e ter idade igual ou superior a 18 anos.

A equipe de coleta de dados foi composta por nove entrevistadores, entre nutricionistas e alunas do curso de graduação em Nutrição da Universidade Federal do Paraná (UFPR). Antes do início da coleta, os entrevistadores receberam treinamento sobre as etapas da pesquisa. O estudo piloto ocorreu, também, alguns meses antes do início da pesquisa, em uma das USF participantes, com gestantes no segundo e terceiro trimestres da gestação. O instrumento utilizado na coleta de dados foi previamente testado e précodificado. Avaliou-se a duração da entrevista e a compreensão das perguntas por parte das entrevistadas. As gestantes participantes da fase de teste do instrumento não foram incluídas na amostra.

As entrevistas ocorreram nas salas de espera da consulta médica de rotina do pré-natal, após acolhimento da gestante e realização da pré-consulta pela equipe de saúde da USF (aferição pressão arterial e peso atual).

Dentre as variáveis demográficas investigadas estiveram: faixa etária (até $20 ; 20-34 ; \geq 35$ ) e coabitação com companheiro (não, sim). Entre as variáveis socioeconômicas avaliadas: escolaridade (até 7; 8-10; $\geq 11$ ) e trabalho remunerado (não, sim). O tabagismo atual foi o comportamento relacionado à saúde investigado (não, sim). Questionados, os antecedentes obstétricos compreenderam: número de gestações $(1 ; 2 ; \geq 3)$. E, por fim, a variável ligada à experiência prévia com amamentação: tempo de amamentação na primeira gestação em meses $(<6 ; 6-23 ; \geq 24)$. As gestantes foram questionadas em relação à IA (não, sim), e, quando a resposta era positiva, a expectativa de duração da amamentação era investigada pelas pesquisadoras. Após coleta, a duração da amamentação registrada como variável contínua em meses foi dividida em três categorias: $0-5$; 6-23; e 24 ou mais. A categoria de referência nas análises foi de 6 a 23 meses.

A dupla entrada de dados foi realizada no programa EpiData ${ }^{\circledR}$ versão $3.0^{14}$, no qual foram inseridos controles para entrada dos campos. Efetuou-se análise descritiva dos dados com cálculo das médias, desvios-padrão, valores medianos, mínimo e máximo para as variáveis contínuas. As variáveis categóricas foram descritas por meio das frequências absolutas (n) e relativas (\%).

Associações entre os desfechos e as variáveis de exposição foram investigadas por meio do teste do Qui-Quadrado de Pearson. A regressão logística multinomial foi empregada para as análises brutas e ajustadas, sendo a categoria de 6 a 23 meses de IA considerada de referência. As análises geraram Odds Ratios (OR) relativas à categoria de referência, e respectivos Intervalos de Confiança de 95\% (IC95\%).

As variáveis que obtiveram níveis de significância com valores de $p$ menores ou iguais a 0,25 na análise de associação com o desfecho foram inseridas na análise ajustada. Inicialmente, foram inseridas as variáveis demográficas, em seguida as socioeconômicas, as de comportamentos relacionados à saúde, de antecedentes obstétricos e de experiência prévia com amamentação. Foram mantidas, nos modelos, as variáveis que na análise ajustada apresentaram valores de p até 0,25 , e consideradas significativas quando valor de $\mathrm{p}$ menor ou igual a 0,05 .

Esta pesquisa foi aprovada pelo Comitê de Ética em Pesquisa em Seres Humanos do Setor de Ciências da Saúde da Universidade Federal do Paraná (UFPR) e foi conduzida dentro dos padrões éticos exigidos por este comitê. Todas as gestantes participantes do estudo assinaram o Termo de Consentimento Livre e Esclarecido (TCLE).

\section{Resultados}

Das 322 gestantes, que atendiam aos critérios de inclusão, convidadas a participar do estudo, 316 completaram a pesquisa, 315 responderam sobre IA, e 286 informaram o tempo de IA. A média de idade das gestantes foi de 26,2 anos (desvio padrão $6,0)$ e variou de 18,0 a 45,7 anos. Com relação ao tempo da amamentação pretendido pelas gestantes com IA $(\mathrm{n}=313)$, a média foi de 13,5 meses $(0$ a 48 meses; IC95\% 12,5; 14,4).

Na Tabela 1, é apresentada a distribuição da amostra de acordo com as características estudadas para as mulheres que indicaram tempo de IA ( $\mathrm{n}=286)$. Observou-se que $75,9 \%$ das gestantes possuíam idade entre 20 e 34 anos, 83,9\% possuíam companheiro, 39,2\% apresentaram entre 8 e 10 anos de escolaridade 57,3\% não possuíam traba- 
Tabela 1. Distribuição das gestantes de acordo com variáveis demográficas, socioeconômicas, comportamentos relacionados com a saúde e antecedentes obstétricos. Colombo-PR, 2016.

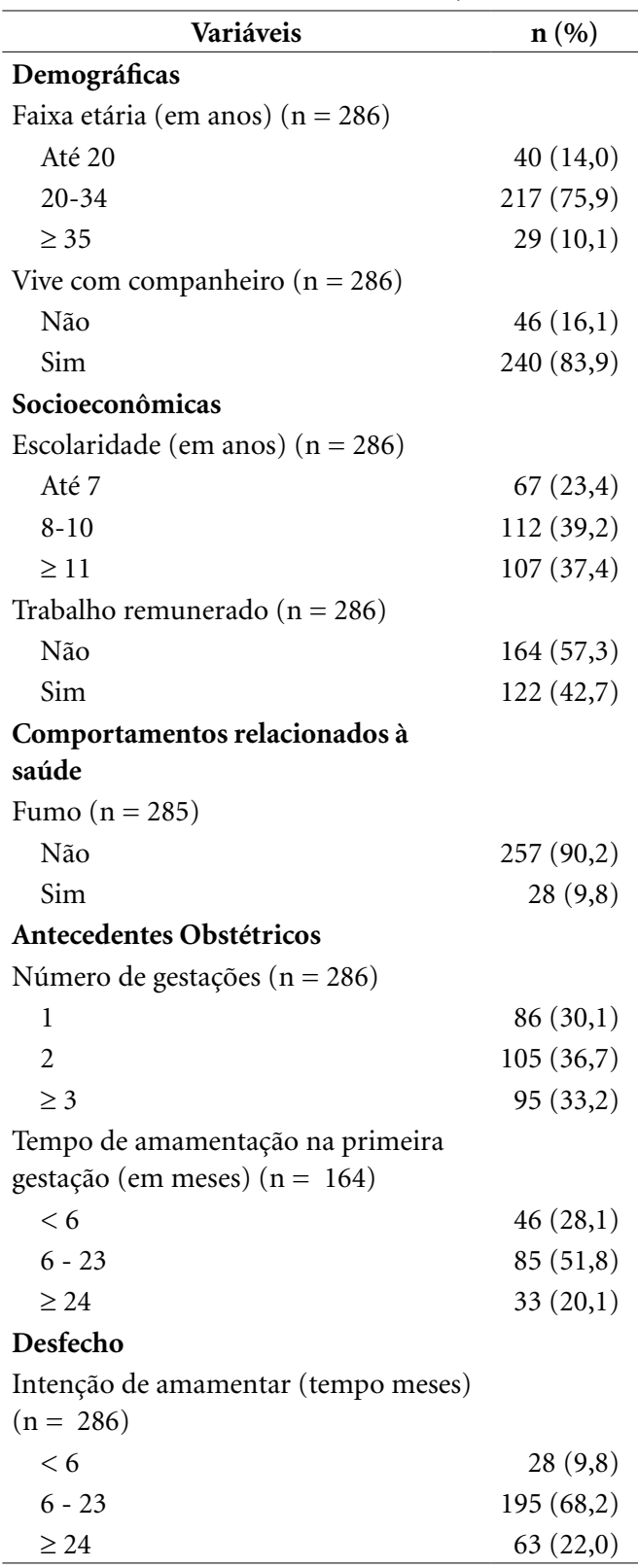

lho remunerado. Encontrou-se, ainda, que 30,1\% eram primíparas, $9,8 \%$ tabagistas, e $20,1 \%$ referiram experiência prévia com amamentação do primeiro filho por 24 meses ou mais. Apenas três $(0,9 \%)$ gestantes declararam não apresentar IA. A IA por menos de 6 e mais de 24 meses foi referida por $9,8 \%$ e $22,0 \%$ das gestantes, respectivamente.

$\mathrm{Na}$ análise não ajustada, observou-se que as chances de amamentar por tempo inferior a 6 meses foram maiores para as gestantes que: declararam não possuir companheiro, ter trabalho remunerado e serem fumantes (Tabela 2). Após a análise ajustada, observou-se que as gestantes que referiram não possuir companheiro (OR $3,23$ IC $95 \% 1,31 ; 7,94)$, as que realizavam atividade remunerada (OR 5,56 IC95\% 2,10; 14,71) e as que eram fumantes (OR 7,79 IC95\% 2,35; 25,81 ) apresentavam maiores chances de IA por um tempo inferior a 6 meses. As gestantes com maior escolaridade informaram com menor frequência IA por tempo inferior a 6 meses, e a associação com maior número de gestações deixou de ser estatisticamente significativa depois do ajuste para as variáveis demográficas e socioeconômicas investigadas (Tabela 3 ).

Por outro lado, a chance de referir IA por tempo igual ou maior a 24 meses apresentou frequência superior entre as gestantes com idade entre 20 e 34 anos, não fumantes, e com histórico anterior de amamentação por período igual ou maior a 24 meses. Após a análise ajustada, verificou-se que as gestantes com idade entre $20 \mathrm{e}$ 34 anos apresentaram chance 3,05 vezes maior (IC95\% 1,02; 9,03) de IA por um período igual ou maior a 24 meses, quando comparadas àquelas com idade inferior a 20 anos. As mulheres que amamentaram por um período igual ou superior a 24 meses em sua primeira gestação apresentaram 7,32 vezes mais chances (IC95\% 1,99; 26,90) de IA prolongada, quando comparadas àquelas que referiram ter amamentado 6 meses ou menos o primeiro filho (Tabela 3 ).

\section{Discussão}

A decisão de amamentar é tomada pela mulher antes do nascimento da criança, isto é, antes ou durante a gravidez, e está relacionada com a duração da amamentação ${ }^{15}$. Este estudo permitiu identificar as características associadas à IA e a duração pretendida entre gestantes, em amostra representativa das gestantes adultas, usuárias do SUS, em acompanhamento pré-natal nas USF de um município da região metropolitana de Curitiba (PR).

A duração média do tempo de IA entre as gestantes foi superior a um ano, sendo que apenas três $(0,9 \%)$ referiram não ter IA. A IA por 24 ou mais meses foi relatada com maior frequência por gestantes de 20 a 34 anos de idade, e que referiram ter amamentado por tempo superior a 24 meses na primeira gestação. De outro lado, a IA por tempo inferior a 6 meses, ou seja, desmame 
Tabela 2. Distribuição das variáveis demográficas, socioeconômicas, comportamentos relacionados à saúde e antecedentes obstétricos de gestantes de acordo com o tempo de intenção de amamentar. Colombo-PR. ( $\mathrm{N}=$ 286).

\begin{tabular}{|c|c|c|c|c|}
\hline \multirow[t]{2}{*}{ Variáveis } & \multirow{2}{*}{$\begin{array}{c}\begin{array}{c}\text { Menos } 6 \\
\text { meses }\end{array} \\
n(\%)\end{array}$} & \multirow{2}{*}{$\begin{array}{c}6 \text { a } 23 \\
\text { meses }\end{array}$} & \multirow{2}{*}{$\begin{array}{c}\begin{array}{c}24 \\
\text { meses }\end{array} \\
\text { ou mais } \\
\mathrm{n}(\%)\end{array}$} & \multirow[b]{2}{*}{$\begin{array}{l}\text { Valor } \\
\text { de } p^{*}\end{array}$} \\
\hline & & & & \\
\hline \multicolumn{5}{|l|}{ Demográficas } \\
\hline Faixa etária $(\mathrm{em}$ anos $)(\mathrm{n}=286)$ & & & & 0,300 \\
\hline Até 20 & $4(10,0)$ & $32(80,0)$ & $4(10,0)$ & \\
\hline $20-34$ & $21(9,7)$ & $142(65,4)$ & $54(24,9)$ & \\
\hline$\geq 35$ & $3(10,3)$ & $21(72,4)$ & $5(17,2)$ & \\
\hline Vive com companheiro $(\mathrm{n}=286)$ & & & & 0,026 \\
\hline Não & $9(19,6)$ & $25(54,3)$ & $12(26,1)$ & \\
\hline Sim & $19(7,9)$ & $170(70,8)$ & $51(21,3)$ & \\
\hline \multicolumn{5}{|l|}{ Socioeconômica } \\
\hline Escolaridade $(\mathrm{em}$ anos $)(\mathrm{n}=286)$ & & & & 0,248 \\
\hline Até 7 & $9(13,4)$ & $40(59,7)$ & $18(26,9)$ & \\
\hline $8-10$ & $11(9,8)$ & $74(66,1)$ & $27(24,1)$ & \\
\hline$\geq 11$ & $8(7,5)$ & $81(75,7)$ & $18(16,8)$ & \\
\hline Trabalho remunerado $(\mathrm{n}=286)$ & & & & 0,001 \\
\hline Não & $7(4,3)$ & $114(69,5)$ & $43(26,2)$ & \\
\hline Sim & $21(17,2)$ & $81(66,4)$ & $20(16,4)$ & \\
\hline \multicolumn{5}{|l|}{ Comportamentos relacionados à saúde } \\
\hline Fumo atual $(\mathrm{n}=285)$ & & & & 0,002 \\
\hline Não & $20(7,8)$ & $179(69,6)$ & $58(22,6)$ & \\
\hline Sim & $8(28,6)$ & $16(57,1)$ & $4(14,3)$ & \\
\hline \multicolumn{5}{|l|}{ Antecedentes Obstétricos } \\
\hline Número de gestações $(\mathrm{n}=286)$ & & & & 0,162 \\
\hline 1 & $4(4,7)$ & $66(76,7)$ & $16(18,6)$ & \\
\hline 2 & $11(10,5)$ & $71(67,6)$ & $23(21,9)$ & \\
\hline$\geq 3$ & $13(13,7)$ & $58(61,0)$ & $24(25,3)$ & \\
\hline Tempo de amamentação primeira gestação (em meses) $(\mathrm{n}=149)$ & & & & 0,001 \\
\hline$<6$ & $10(24,4)$ & $26(63,4)$ & $5(12,2)$ & \\
\hline$>6-23$ & $4(5,2)$ & $59(76,6)$ & $14(18,2)$ & \\
\hline$\geq 24$ & $4(12,9)$ & $10(32,3)$ & $17(54,8)$ & \\
\hline
\end{tabular}

precoce, foi mais frequentemente relatada entre as gestantes que não possuíam companheiro, realizavam atividade remunerada e eram fumantes.

A mediana do tempo de AME no Brasil, entre 1999 e 2008 , era de 54,1 dias (1,8 meses) e de aleitamento materno era de 341,6 dias (aproximadamente 11,2 meses $)^{7}$. Esta diferença positiva observada entre estudos de IA e estudos de prevalência de aleitamento materno pode ser atribuída ao fato de que o presente estudo investigou a intenção de realizar a amamentação, diferente- mente dos estudos que avaliaram a prevalência do AME aos seis meses ${ }^{16,17}$ e após os 12 meses $^{18}$, ou seja, a efetivação prática do comportamento.

A diferença entre a intenção da amamentação da gestante e a efetivação enquanto nutriz pode ocorrer devido a fatores não necessariamente controlados de forma direta pela mulher, pois a amamentação está sujeita a determinantes fisiológicos (leite insuficiente, cansaço, fadiga), clínicos (mastite, ingurgitamento mamário) e culturais, além da participação da criança, das 
Tabela 3. Associação da intenção de amamentar de gestante e variáveis demográficas, socioeconômicas, comportamentos relacionados à saúde e antecedentes obstétricos. Colombo-PR, 2016.

\begin{tabular}{|c|c|c|c|c|}
\hline \multirow{2}{*}{ Variáveis } & \multicolumn{2}{|c|}{$\begin{array}{c}\text { Menos de } 6 \text { meses versus } \\
6 \text { a } 23 \text { meses }\end{array}$} & \multicolumn{2}{|c|}{$\begin{array}{c}24 \text { ou mais meses versus } \\
6 \text { a } 23 \text { meses } \\
\end{array}$} \\
\hline & OR (IC95\%) & $\begin{array}{l}\text { OR Ajustada } \\
\text { (IC95\%) }\end{array}$ & OR (IC95\%) & $\begin{array}{l}\text { OR Ajustada } \\
\text { (IC95\%) }\end{array}$ \\
\hline \multicolumn{5}{|l|}{ Demográficas } \\
\hline \multicolumn{5}{|c|}{ Faixa etária em anos $(\mathrm{n}=286)$} \\
\hline Até 20 & 1 & 1 & 1 & 1 \\
\hline $20-34$ & $1,18(0,37 ; 3,68)$ & $1,19(0,38 ; 3,75)$ & $3,04(1,02 ; 9,00)$ & $3,05(1,02 ; 9,03)$ \\
\hline$\geq 35$ & $1,14(0,23 ; 5,63)$ & $1,06(0,21 ; 5,35)$ & $1,90(0,45 ; 7,92)$ & $1,86(0,44 ; 7,75)$ \\
\hline Valor de $\mathrm{P}^{\star}$ & 0,834 & $0,896^{\mathrm{a}}$ & 0,309 & $0,328^{\mathrm{a}}$ \\
\hline \multicolumn{5}{|c|}{ Vive com companheiro $(\mathrm{n}=286)$} \\
\hline Sim & 1 & 1 & 1 & 1 \\
\hline Não & $3,22(1,31 ; 7,90)$ & $3,23(1,31 ; 7,94)$ & $1,60(0,75 ; 3,40)$ & $1,62(0,75 ; 3,49)$ \\
\hline Valor de $\mathrm{P}^{\star}$ & 0,011 & $0,011^{\mathrm{a}}$ & 0,223 & $0,237^{\mathrm{a}}$ \\
\hline \multicolumn{5}{|l|}{ Socioeconômicas } \\
\hline \multicolumn{5}{|c|}{ Escolaridade $(\mathrm{em}$ anos $)(\mathrm{n}=286)$} \\
\hline Até 7 & 1 & 1 & 1 & 1 \\
\hline $8-10$ & $0,66(0,25 ; 1,72)$ & $0,64(0,23 ; 1,80)$ & $0,81(0,40 ; 1,64)$ & $0,89(0,43 ; 1,85)$ \\
\hline$\geq 11$ & $0,43(0,16 ; 1,22)$ & $0,29(0,09 ; 0,86)$ & $0,50(0,23 ; 1,05)$ & $0,50(0,23 ; 1,10)$ \\
\hline Valor de $\mathrm{P}^{\star}$ & 0,113 & $0,023^{\mathrm{b}}$ & 0,059 & $0,074^{\mathrm{b}}$ \\
\hline \multicolumn{5}{|c|}{ Trabalho remunerado $(\mathrm{n}=286)$} \\
\hline Não & 1 & 1 & 1 & 1 \\
\hline Sim & $4,22(1,71 ; 10,40)$ & $5,56(2,10 ; 14,71)$ & $0,65(0,36 ; 1,19)$ & $0,66(0,35 ; 1,24)$ \\
\hline Valor de $\mathrm{P}^{\star}$ & 0,002 & $0,001^{\mathrm{b}}$ & 0,168 & $0,253^{\mathrm{b}}$ \\
\hline \multicolumn{5}{|c|}{$\begin{array}{l}\text { Comportamentos relacionados } \\
\text { à saúde }\end{array}$} \\
\hline \multicolumn{5}{|l|}{ Fumo atual $(\mathrm{n}=285)$} \\
\hline Não & 1 & 1 & 1 & 1 \\
\hline Sim & $4,4(1,70 ; 11,76)$ & $7,79(2,35 ; 25,81)$ & $0,77(0,25 ; 2,40)$ & $0,54(0,16 ; 1,75)$ \\
\hline Valor de P & 0,002 & 0,001 & 0,654 & 0,301 \\
\hline \multicolumn{5}{|c|}{ Antecedentes Obstétricos } \\
\hline \multicolumn{5}{|c|}{ Número de gestações $(\mathrm{n}=285)$} \\
\hline 1 & 1 & 1 & 1 & 1 \\
\hline 2 & $2,56(0,77 ; 8,42)$ & $2,71(0,77 ; 9,57)$ & $1,34(0,65 ; 2,75)$ & $1,25(0,59 ; 2,63)$ \\
\hline$\geq 3$ & $3,70(1,14 ; 11,97)$ & $3,44(0,86 ; 13,75)$ & $1,70(0,83 ; 3,52)$ & $1,22(0,54 ; 2,79)$ \\
\hline Valor de $\mathrm{P}^{\star}$ & 0,027 & $0,122^{\mathrm{d}}$ & 0,145 & $0,556^{\mathrm{d}}$ \\
\hline \multicolumn{5}{|c|}{ Experiência com amamentação } \\
\hline \multicolumn{5}{|c|}{$\begin{array}{l}\text { Tempo de amamentação na } \\
\text { primeira gestação (em meses) ( } \mathrm{n} \\
=164)\end{array}$} \\
\hline$<6$ & 1 & 1 & 1 & 1 \\
\hline $6-23$ & $0,18(0,50 ; 0,61)$ & $0,08(0,02 ; 0,39)$ & $1,23(0,40 ; 3,78)$ & $1,14(0,36 ; 3,66)$ \\
\hline$\geq 24$ & $1,04(0,30 ; 4,09)$ & $0,46(0,08 ; 2,68)$ & $8,84(2,57 ; 30,40)$ & $7,32(1,99 ; 26,90)$ \\
\hline Valor de $\mathrm{P}^{\star}$ & 0,333 & $0,080^{\mathrm{e}}$ & $<0,001$ & $0,003^{\mathrm{e}}$ \\
\hline \multicolumn{5}{|c|}{$\begin{array}{l}\text { Comportamentos relacionados } \\
\text { à saúde }\end{array}$} \\
\hline \multicolumn{5}{|l|}{ Fumo atual $(\mathrm{n}=285)$} \\
\hline Não & 1 & 1 & 1 & 1 \\
\hline Sim & $4,4(1,70 ; 11,76)$ & $7,79(2,35 ; 25,81)$ & $0,77(0,25 ; 2,40)$ & $0,54(0,16 ; 1,75)$ \\
\hline Valor de P & 0,002 & 0,001 & 0,654 & 0,301 \\
\hline
\end{tabular}


Tabela 3. Associação da intenção de amamentar de gestante e variáveis demográficas, socioeconômicas, comportamentos relacionados à saúde e antecedentes obstétricos. Colombo-PR, 2016.

\begin{tabular}{|c|c|c|c|c|}
\hline \multirow{2}{*}{ Variáveis } & \multicolumn{2}{|c|}{$\begin{array}{c}\text { Menos de } 6 \text { meses versus } \\
6 \text { a } 23 \text { meses }\end{array}$} & \multicolumn{2}{|c|}{$\begin{array}{c}24 \text { ou mais meses versus } \\
6 \text { a } 23 \text { meses } \\
\end{array}$} \\
\hline & OR (IC95\%) & $\begin{array}{l}\text { OR Ajustada } \\
\text { (IC95\%) }\end{array}$ & OR (IC95\%) & $\begin{array}{l}\text { OR Ajustada } \\
\text { (IC95\%) }\end{array}$ \\
\hline \multicolumn{5}{|c|}{ Antecedentes Obstétricos } \\
\hline \multicolumn{5}{|c|}{ Número de gestações $(\mathrm{n}=285)$} \\
\hline 1 & 1 & 1 & 1 & 1 \\
\hline 2 & $2,56(0,77 ; 8,42)$ & $2,71(0,77 ; 9,57)$ & $1,34(0,65 ; 2,75)$ & $1,25(0,59 ; 2,63)$ \\
\hline$\geq 3$ & $3,70(1,14 ; 11,97)$ & $3,44(0,86 ; 13,75)$ & $1,70(0,83 ; 3,52)$ & $1,22(0,54 ; 2,79)$ \\
\hline Valor de $\mathrm{P}^{\star}$ & 0,027 & $0,122^{\mathrm{d}}$ & 0,145 & $0,556^{\mathrm{c}}$ \\
\hline \multicolumn{5}{|c|}{ Experiência com amamentação } \\
\hline \multicolumn{5}{|c|}{$\begin{array}{l}\text { Tempo de amamentação na } \\
\text { primeira gestação (em meses) ( } \mathrm{n} \\
=164)\end{array}$} \\
\hline$<6$ & 1 & 1 & 1 & 1 \\
\hline $6-23$ & $0,18(0,50 ; 0,61)$ & $0,08(0,02 ; 0,39)$ & $1,23(0,40 ; 3,78)$ & $1,14(0,36 ; 3,66)$ \\
\hline$\geq 24$ & $1,04(0,30 ; 4,09)$ & $0,46(0,08 ; 2,68)$ & $8,84(2,57 ; 30,40)$ & $7,32(1,99 ; 26,90)$ \\
\hline Valor de $\mathrm{P}^{*}$ & 0,333 & $0,080^{\mathrm{e}}$ & $<0,001$ & $0,003^{\mathrm{e}}$ \\
\hline
\end{tabular}

pressões do ambiente, rede de apoio e de aspectos psicológicos $^{19-21}$.

Apesar de a intenção representar uma expectativa em relação a um comportamento futuro, os resultados encontrados nesta pesquisa são consistentes com achados provenientes de outros estudos sobre os fatores que influenciam na $\mathrm{IA}^{9-}$ ${ }^{11,19}$, mas apresentando uma expectativa superior em relação a pesquisas realizadas nos Estados Unidos $(44,9 \% \text { a } 64,6 \%)^{9,11,15,22,23}$, na Inglaterra $(20 \%)^{24}$ e na China $(53,9 \%)^{25}$. Além disso, os dados disponíveis dos estudos com abrangência nacional sobre aleitamento materno são de $2008^{7}$, e estudos que avaliaram a tendência temporal na duração desse aleitamento, no Brasil, têm indicado tendência de aumento na duração total, e na prevalência de $\mathrm{AME}^{16,17,26}$.

Além de contribuir na duração total de aleitamento materno ${ }^{9}$, possuir um companheiro pode influenciar positivamente na IA, como encontrado neste e em outros estudos ${ }^{15,24,27}$. Durante o período de aleitamento materno, os amparos emocionais, sociais e econômicos aparecem como os mais importantes, e o companheiro destaca-se e tem maior peso nesse momento ${ }^{10,11,28-30}$. Por outro lado, um estudo retrospectivo com mulheres militares residentes na região metropolitana de
Belo Horizonte (MG) demonstrou que as mulheres casadas amamentaram por tempo inferior, quando comparadas às solteiras (OR 7,08 IC $95 \% 1,98 ; 25,16)^{31}$.

Uma revisão de literatura indicou sentimentos ambíguos dos companheiros durante a experiência da amamentação, incluindo dificuldade de se sentirem parte do processo de amamentação e queixas sobre perda do interesse sexual de suas companheiras, além de sentimento de exclusão e abandono, nas madrugadas, no período da amamentação $^{32}$. Ações educativas por parte das equipes de saúde com vistas à inclusão do pai no pré-natal, com maiores informações, esclarecimentos das vantagens, de dúvidas e tabus do aleitamento materno podem influenciar positivamente no sentimento de apoio para a gestante na $\mathrm{IA}^{33}$.

As gestantes com maior escolaridade apresentaram menor chance de referir IA por tempo inferior a seis meses. Estudo semelhante nos Estados Unidos encontrou que 59,1\% das mulheres que pretendiam amamentar possuíam ensino médio completo ou eram graduadas ${ }^{25}$. Morgado et $\mathrm{al}^{28}$, em coorte prospectiva que buscou investigar a associação entre rede e apoio social e as práticas alimentares de lactentes por meio de recor- 
datório alimentar com as mães, encontraram que mulheres com ensino fundamental incompleto apresentaram 5 vezes mais chances (IC95\% 1,77; 14,04) de introduzir alimentos sólidos e 4,37 (IC95\% 1,32; 14,5) de não alimentarem seu bebê com leite materno, quando comparadas àquelas com maior escolaridade.

A baixa escolaridade está associada à menor duração do aleitamento materno. Um estudo utilizando dados da Pesquisa Estadual de Saúde e Nutrição de Pernambuco (PESN), de 1991, 1997 e 2006, considerou a maior escolaridade como fator de proteção na duração do AME. Ainda nesse estudo, mulheres com 9 ou mais anos de escolaridade apresentaram maior prevalência de AME ao sexto mês (RP 1,8 IC95\% 1,0;3,4), quando comparadas àquelas com menor escolaridade.

Uma das alternativas para incentivar a prática de aleitamento materno entre as mulheres com baixa escolaridade é a realização de orientações durante o acompanhamento pré-natal. O maior número de consultas no pré-natal pode influenciar positivamente no conhecimento sobre aleitamento materno ${ }^{34}$. Um estudo transversal na Índia, em 2012, encontrou que mulheres que receberam orientações durante o pré-natal a respeito do AME apresentaram 2,68 vezes mais chances (IC $95 \% 1,27 ; 5,65$ ) de possuírem intenção de amamentar exclusivamente até o sexto mês ${ }^{35}$.

A realização de trabalho remunerado, por sua vez, tem sido um dos fatores mais frequentemente citados nos estudos para interrupção precoce do aleitamento materno $0^{36,37}$. Nesta pesquisa, as gestantes que exerciam trabalho remunerado afirmaram ter uma IA por um período inferior a 6 meses 5,56 vezes maior quando comparadas àquelas que não referiram realizar trabalho remunerado. Este resultado diverge daquele identificado por outra pesquisa, a qual encontrou trabalho materno na gestação como fator de proteção ao aleitamento materno ${ }^{25}$; outros estudos sobre IA não encontraram associação entre as variáveis $^{15,26,38}$. Vale ressaltar, que o presente estudo mensurou a IA e sua duração total, diferentemente de outros, os quais avaliaram a IA versus intenção de alimentação artificial $^{19}$. Adicionalmente, é importante destacar que a maioria dos empregadores, não adota a licença maternidade de seis meses, mas, sim, de quatro meses, o que pode ter influenciado as estimativas sobre tempo de IA entre as mulheres avaliadas.

Uma pesquisa conduzida no interior de São Paulo, em 2008, com 200 trabalhadoras, apontou que apenas $23,4 \%$ conseguiam amamentar durante a jornada de trabalho, e que $57 \%$ dessas, com jornada de trabalho inferior a oito horas, conseguiram manter a amamentação por mais de quatro meses. Na mesma pesquisa, evidenciou-se a falta de local adequado para amamentação ou posto de coleta de leite materno, visto que apenas $12,5 \%$ forneciam local apropriado para esses fins $^{37}$. Ainda, quase metade das trabalhadoras brasileiras $(47,9 \%)$ apresentava vínculo informal de trabalho, o que dificulta o acesso ao direito à licença maternidade ${ }^{37}$. Nota-se, nesse sentido, que a licença maternidade ofertada para as trabalhadoras formais é benéfica e favorece a adesão ao aleitamento materno nos primeiros meses do bebê. Contudo, no Brasil, para a manutenção do aleitamento, faz-se necessário que outras condições sejam oferecidas, tais como disponibilidade de creche no local de trabalho, sala para ordenha e estocagem de leite materno, flexibilização da jornada de trabalho e pausas de 30 minutos durante essa jornada ${ }^{31,37}$.

Entre as gestantes fumantes avaliadas no presente estudo, verificou-se chance 7,79 vezes maior de referir intenção de amamentar por tempo inferior a 6 meses, quando comparadas àquelas não fumantes. O uso do cigarro prejudica os estágios da reprodução humana, em especial a gestação, devido às suas repercussões no parto e os desfechos no desenvolvimento e vida pós-uterina do bebê $\hat{e}^{39}$. Durante a gestação, o consumo do cigarro está associado a complicações no período prénatal, como aborto precoce, baixo peso ao nascer, parto prematuro, além de complicações na saúde a longo prazo $^{39,40}$. O tabagismo tem-se mostrado como fator negativo na $\mathrm{IA}^{14,23,25} \mathrm{e}$ na duração total do aleitamento materno ${ }^{23}$.

Em um estudo transversal na Philadelphia, Estados Unidos (EUA), com 2.690 mulheres, entre os anos de 1999 e 2002, verificou-se que apenas $33,1 \%$ das mulheres tabagistas relataram intenção de amamentar. Os autores atribuíram a menor IA relatada entre as gestantes fumantes ao fato de elas preverem não cessar o uso do cigarro após o parto e, por conseguinte, não desejarem expor o bebê à nicotina e outras substâncias tóxicas através do leite materno ${ }^{15}$. Uma revisão sistemática aponta que gestantes tabagistas possivelmente sofrem influência negativa na duração do aleitamento materno, por saberem da gama de malefícios causados pelo cigarro, da dificuldade na cessação do hábito e falta de apoio profissional23.

O tempo de amamentação do primeiro filho apareceu como o fator com maior magnitude de associação na proteção ao aleitamento materno prolongado entre as gestantes avaliadas. Uma pesquisa de Carrascoza et al. ${ }^{40}$ encontrou que mães 
que haviam amamentado prolongadamente apresentaram chances 1,45 maiores de estender o período de amamentação do bebê atual. Meyerink e Marquis $^{39}$ verificaram, entre mulheres pobres no sudoeste dos EUA, que as experiências prévias com amamentação e o fato de a própria mãe ter sido amamentada quando bebê influenciaram positivamente a iniciação e a duração do aleitamento materno. Os autores também verificaram que o efeito da renda e da escolaridade nos padrões de aleitamento materno pode ser influenciado pelas experiências maternas e familiares com amamentação ${ }^{39}$.

Um estudo realizado em Piracicaba (São Paulo) verificou que mulheres que amamentaram seus filhos anteriores por um período superior a 6 meses apresentam maiores chances de prolongar a amamentação do atual bebê, quando comparadas àquelas que nunca haviam amamentado ${ }^{40}$. Takushi et al. ${ }^{41}$, em estudo qualitativo, encontraram como fator de motivação de gestantes para amamentar o novo bebê as experiências prévias com aleitamento. As gestantes atribuíram a saúde do primogênito ao tempo de aleitamento materno.

Dentre as limitações do presente estudo, podem ser destacados seu desenho transversal e a ausência do questionamento sobre a IA, exclusivamente. Além disso, é possível que as gestantes, ao participarem da pesquisa no ambiente da USF, tenham-se sentido constrangidas a responderem de acordo com o comportamento que seria esperado, e informado maior período de IA, especialmente aquelas com maior acesso à informação. Outros fatores não investigados nesta pesquisa podem influenciar a manutenção do AME, até os 6 meses, e sua continuidade além dos 2 anos de vida. Adicionalmente, a amostra pode não ter sido suficiente para detectar associações significativas entre algumas variáveis investigadas e o tempo de IA, sobretudo aquelas de menor magnitude de associação. Estudos futuros podem verificar nessas mães se o tempo de amamentação foi semelhante com a sua intenção. Assim, podem também investigar o processo de decisão sobre a IA a partir da Teoria do Comportamento Plane$\mathrm{jado}^{42}$, e empregar instrumentos que permitam captar o impacto da atitude, normas subjetivas e percepção de controle de comportamento na tomada de decisão sobre IA.

\section{Conclusão}

A prevalência de IA mostrou-se satisfatória, com quase totalidade das gestantes relatando-a
(99,05\%), sendo 90,2\% delas gestantes com IA por 6 ou mais meses. Desse modo, identificar as mulheres com maiores chances de optar por amamentar por menos de 6 meses, ou seja, com intenção de desmamar precocemente, torna-se imprescindível e deve ocorrer por meio de ações efetivas e eficazes na proteção e promoção do aleitamento materno a este grupo mais vulnerável. Ações de saúde podem ser promovidas pelas equipes de saúde da família e por grupos multiprofissionais envolvidos nos cuidados pré-natais, como a utilização de guias práticos da amamentação, cartilhas com os direitos trabalhistas da mulher $\mathrm{e}$ nutriz, demonstrações de aleitamento materno e visita de acompanhamento no puerpério.

Nesse sentido, torna-se válido destacar a importância do acompanhamento pré-natal por equipe multiprofissional para assistência integral à promoção da amamentação, considerando os aspectos sociais e psicoemocionais envolvidos no processo de aleitamento materno, além da importância do compartilhamento de experiências prévias entre as gestantes. O número de consultas de pré-natal e a qualidade das informações transmitidas durante ele, acerca da amamentação, podem aumentar o conhecimento dos benefícios e sanar possíveis dúvidas e receios sobre aleitamento materno.

Os fatores como não possuir companheiro, possuir trabalho remunerado e ser fumante estiveram associados negativamente à duração do aleitamento materno. Ações de identificação destas mulheres no período pré-natal, bem como a implantação de estratégias embasadas em suas características de vulnerabilidade, podem influenciar positivamente na duração do aleitamento materno.

Nota-se, ainda, a importância da ampliação dos benefícios e direitos trabalhistas das gestantes e/ou mães trabalhadoras, tais como expansão da licença maternidade remunerada - de 4 para 6 meses para todas as trabalhadoras formais -, além de políticas que auxiliem as trabalhadoras informais, proporcionando condições favoráveis ao aleitamento materno por mais de 6 meses de duração.

Por fim, a experiência prévia com aleitamento materno por tempo maior ou igual a 24 meses do primeiro filho mostrou-se como fator de proteção para uma IA prolongada das gestantes participantes da pesquisa. A criação de ações que permitam troca de conhecimentos, saberes e experiências entre mulheres que tiveram experiências positivas e duradouras em amamentações anteriores com primíparas pode auxiliar 
na intenção de amamentar de mulheres com experiências anteriores breves ou negativas. Dessa forma, o presente trabalho oferece informações relevantes para o campo da saúde coletiva, dada a existência de informações incipientes acerca da IA no meio científico e que podem ser empregadas no planejamento das ações na atenção primária à saúde e na identificação de grupos com maior vulnerabilidade ao desmame precoce.

* Artigo parte integrante do trabalho de conclusão de curso de Fernandes, RC, intitulado "Intenção de Amamentar em Gestantes de Unidades de Saúde da Família”, na Residência Multiprofissional em Saúde da Família, da Universidade Federal do Paraná, apresentado em abril de 2017.

\section{Colaboradores}

RC Fernandes e DA Höfelmann participaram do planejamento da pesquisa, participou da análise e interpretação dos dados, da redação do artigo, e aprovação da versão final do artigo.

\section{Agradecimentos}

Os autores gostariam de agradecer ao Centro de Assessoria em Publicações Acadêmicas (CAPA www.capa.ufpr.br) da Universidade Federal do Paraná pela assistência na edição de língua inglesa. 


\section{Referências}

1. Brasil. Ministério da Saúde (MS). Saúde da criança: Aleitamento materno e alimentação complementar. Brasília: MS; 2015.

2. Horta BL, Mola CL, Victora CG. Long-term consequences of breastfeeding on cholesterol, obesity, systolic blood pressure and type 2 diabetes: a systematic review and meta-analysis. Acta Paediatr 2015; 104(467):30-37.

3. World Health Organization (WHO). Infant and Young Child Feeding: Model Chapter for Textbooks for Medical Students and Allied Health Professionals. Geneva: WHO; 2009.

4. World Health Organization (WHO). The Global strategy for infant and Young child feeding. Geneva: WHO; 2003.

5. Kramer MS, Kakuma R. The optimal duration of exclusive breastfeeding: a systematic review. Geneva: World Health Organization (WHO); 2001.

6. Victora CG, Bahl R, Barros AJD, França GVA, Horton S, Krasevec J, Murch S, Sankar MJ, Walker N, Rollins NC; Lancet Breastfeeding Series Group. Breastfeeding in the 21st century: Epidemiology, mechanisms, and lifelong effect. Lancet 2016; 387(10017):475-490.

7. Brasil. Ministério da Saúde (MS). II Pesquisa de Prevalência de Aleitamento Materno nas Capitais Brasileiras e Distrito Federal. Brasília: MS; 2009.

8. Brasil. Ministério da Saúde (MS). Pesquisa Nacional de Demografia e Saúde da Criança e da Mulher - PNDS 2006: dimensões do processo reprodutivo e da saúde da criança. Brasília: MS; 2009.

9. Arora S, McJunkin C, Wehrer J, Kuhn P. Major factors influencing breastfeeding rates: mother's perception of father's attitude and milk supply. Pediatrics 2000; 106(5):E67.

10. Faleiros FTV, Trezza EMC, Carandina L. Aleitamento materno: fatores de influência na sua decisão e duração. Rev. Nutr. 2006; 19(5):623-630.

11. Vieira TO, Martins CC, Santana GS, Vieira GO, Silva LR. Intenção materna de amamentar: revisão sistemática. Cien Saude Colet 2016; 21(12):3845-3858.

12. Instituto Paranaense de Desenvolvimento Econômico e Social (IPARDES). Caderno Estatístico Município de Colombo. Curitiba: IPARDES; 2016.

13. Lauritsen JM. EpiData Data Entry, Data Management and basic Statistical Analysis System. Odense: EpiData Association; 2000-2006.

14. Kwak C, Clayton-Matthews A. Multinomial Logistic Regression. Nursing Research 2002; 51(6):404-410.

15. Lee HJ, Rubio MR, Elo IT, McCollum KF, Chung EK, Culhane JF. Factors associated with intention to breastfeed among low-income, inner-city pregnant women. Matern Child Health J 2005; 9(3):253-261.

16. Faleiros JJ, Kalil G, Casarin DP, Laque Júnior PA, Santos IS. Avaliação do impacto de um programa de puericultura na promoção da amamentação exclusiva. Cad Saude Publica 2005; 21(2):482-489.

17. Marques RFSV, Lopez FA, Braga JAP. O crescimento de crianças alimentadas com leite materno exclusivo nos primeiros 6 meses de vida. Rev Chil Pediatr 2006; 77(5):529-530.
18. Carrascoza KC, Costa Junior AL, Ambrosano GM, Moraes AB. Prolongamento da amamentação após o primeiro ano de vida: argumentos das mães. Psic Teor Pesq 2005; 21(3):271-277.

19. Cabieses B, Waiblinger D, Santorelli G, McEachan RR. What factors explain pregnant women's feeding intentions in Bradford, England: a multi-methods, multi-ethnic study. BMC Pregnancy Childbirth 2014; 14:50.

20. Gijsbers B, Mesters I, Knottnerus JA, Van Schayck CP. Factors associated with the initiation of breastfeeding in asthmatic families: the attitude-social influence-self-efficacy model. Breastfeeding Medicine 2006; 1(4):236-246.

21. Saffari M, Pakpour AH, Chen H. Factors influencing exclusive breastfeeding among Iranian mothers: a longitudinal population-based study. Health Promot Perspect 2017; 7(1):34-41.

22. Di Girolamo A, Thompson N, Martorell R, Fein S, Grummer-Strawn L. Intention or experience? Predictors of continued breastfeeding. Health Educ Behav 2005; 32(2):208-226.

23. Humphreys AS, Thompson NJ, Miner KR. Intention to breastfeed in low-income pregnant women: the role of social support and previous experience. Birth 1998; 25(3):169-174.

24. Mclnnes R, Love J, Stone D. Independent predictors of breastfeeding intention in a disadvantaged population of pregnant women. BMC Public Health 2001; 1(1):10-13.

25. Lau Y. Breastfeeding intention among pregnant Hong Kong chinese women. Matern Child Health J 2009; 14(5):790-798.

26. Boccolini CS, Carvalho ML, Oliveira MIC. Fatores associados ao aleitamento materno exclusivo nos primeiros seis meses de vida no Brasil: revisão sistemática. Rev Saude Publica 2015; 49:91.

27. Alexander A, O'Riordan MA, Furman L. Do breastfeeding intentions of pregnant inner-city teens and adult women differ? Breastfeed Med 2010; 5(6):289-296.

28. Morgado CMC, Werneck GL, Hasselmann MH. Rede e apoio social e práticas alimentares de crianças no quarto mês de vida. Cien Saude Colet 2013; 18(2):367376.

29. Niquini RP, Bittencourt SA, Lacerda EMA, Leal MC. Fatores associados à introdução precoce de leite artificial, Município do Rio de Janeiro, 2007. Rev Bras Epidemiol 2009; 12(3):446-457.

30. Rea MF, Venâncio SI, Batista LE, Santos RG, Greiner T. Possibilidades e limitações da amamentação entre mulheres trabalhadoras formais. Rev Saude Publica 1997; 31(2):149-156.

31. Brasileiro AA, Ambrosano GMB, Marba STM, Possobon RF. A amamentação entre filhos de mulheres trabalhadoras. Rev Saude Publica 2012; 46(4):642-648.

32. Marques ES, Cotta RMM, Magalhães KA, Sant'Ana LFR, Gomes AP, Batista RS. A influência da rede social da nutriz no aleitamento materno: o papel estratégico dos familiares e dos profissionais de saúde. Cien Saude Colet 2010; 15(1):1391-1400. 
33. Caminha MFC, Batista FM, Serva VB, Arruda IKG, Figueiroa JN, Lira PIC. Tendências temporais e fatores associados à duração do aleitamento materno em Pernambuco. Rev Saude Publica 2010; 44(2):240-248.

34. Escobar AM, Ogawa AR, Hiratsuka M, Kawashita MY, Teruya PY, Grisi S, Tomikawa SO. Breastfeeding and socioeconomic cultural status: factors that lead to early weaning. Rev Bras Saude Mater Infant 2002; 2(3):253-261.

35. Behera D, Anil Kumar K. Os preditores da intenção de amamentar exclusivamente entre mulheres grávidas rurais na Índia: um estudo usando a teoria do comportamento planejado. Saúde Rural e Remota 2015; 15(3):3405.

36. Freitas TCSB, Silva SC, Chaves RG, Lamounier JA. Prevalência do aleitamento materno e fatores associados à interrupção da amamentação em mulheres militares. Rev Paul Pediatr 2012; 30(4):493-498.

37. Araujo AMC, Lombardi MR. Trabalho informal, gênero e raça no Brasil do início do século XXI. Cad Pesqui 2013; 43(149):452-477.

38. Insaf TZ, Fortner RT, Pekow P, Dole N, Markenson G, Chasan-Taber L. Prenatal stress, anxiety, and depressive symptoms as predictors of intention to breastfeed among Hispanic women. J Womens Health 2011; 20(8):1183-1192.

39. Meyerink RO, Marquis GS. Breastfeeding initiation and duration among low-income women in Alabama: the importance of personal and familial experiences in making infant-feeding choices. Journal of Human Lactation 2002; 18(1):38-44.

40. Carrascoza KC, Costa Júnior AL, Moraes AL. Fatores que influenciam o desmame precoce e a extensão do aleitamento materno. Estud Psicol 2005; 22(4):433440.

41. Takushi SAM, Tanaka AC, d'Andretta, Gallo PR, Machado MAMP. Motivação de gestantes para o aleitamento materno. Rev Nutr 2008; 21(5):491-502.

42. Ajzen I. The Theory of Planned Behavior. Organizational Behavior And Human Decision Processes 1991; 50(2):179-211.

Artigo apresentado em 23/07/2017

Aprovado em 19/07/2018

Versão final apresentada em 21/07/2018 